\title{
Numerical evidence for cloud droplet nucleation at the cloud-environment interface
}

\author{
J. Sun ${ }^{1,2}$, H. Leighton ${ }^{2}$, M. K. Yau ${ }^{2}$, and P. Ariya ${ }^{2,3}$ \\ ${ }^{1}$ Key Laboratory of Cloud-Precipitation Physics and Severe Storms (LACS), Institute of Atmospheric Physics, Chinese \\ Academy of Sciences, Beijing 100029, China \\ ${ }^{2}$ Department of Atmospheric and Oceanic Sciences, McGill University, Montreal, Quebec H3A 0B9, Canada \\ ${ }^{3}$ Department of Chemistry, McGill University, Montreal, Quebec H3A 2K6, Canada
}

Correspondence to: J. Sun (Jimings@mail.iap.ac.cn)

Received: 23 June 2012 - Published in Atmos. Chem. Phys. Discuss.: 18 July 2012

Revised: 17 December 2012 - Accepted: 19 December 2012 - Published: 21 December 2012

\begin{abstract}
Cumulus clouds have long been recognized as being the results of ascending moist air from below the cloud base. Cloud droplet nucleation is understood to take place near the cloud base and inside accelerating rising cloudy air. Here we describe circumstances under which cloud droplet nucleation takes place at the interface of ascending cloudy air and clear air. Evaporation is normally expected to occur at this interface. However, continuity of moving air requires cloud-free air above the boundary of rising cloudy air to move upwards in response to the gradient force of perturbation pressure. We used a one and half dimensional nonhydrostatic cloud model and the Weather Research and Forecast model to investigate the impacts of this force on the evolution of cloud spectra. Our study shows that expansion and cooling of ascending moist air above the cloud top causes it to become supersaturated with condensation rather than evaporation occurring at the interface. We also confirm that Eulerian models can describe the cloud droplet activation and prohibit spurious activation at this interface. The continuous feeding of newly activated cloud droplets at the cloud summit may accelerate warm rain formation.
\end{abstract}

\section{Introduction}

Cumulus clouds result from the ascent of moist air parcels. An unresolved issue in cloud physics is why observed cumulus cloud droplet spectra even in the core of cumulus clouds are broader than the spectra predicted by cloud droplet nucleation and condensational growth in adiabatically ascending parcels (Pruppacher and Klett, 1997). The cloud droplet size distribution will influence the rate of formation of precipitation and the cloud lifetime. Typically, the evolution of droplet spectra in parcel models is only influenced by the thermodynamic properties of moist air from below the cloud base and the thermodynamic properties of the environmental air is ignored. However, the most important property of the atmosphere is the air continuity. Moving air must obey the air continuity constraint. In the developing stage of cumulus clouds, the ascending cloud air must exert a force on the cloud-free air above the cloud air so that cloud-free air can move away from the path of the cloud air. Parcel theory assumes that the pressure of ascending parcels adjusts to their ambient pressure simultaneously. This assumption means that cloud free air above cloud top can horizontally move away from the cloud path without any forces when cloud air moves upwards. The thermodynamic properties of the environmental air are unchanged. Any studies with Eulerian models that invoke this hypothesis may give the misleading conclusion that evaporation necessarily occurs at the cloud-environment interface of cumulus clouds. In other words, the top interface of any ascending air from near the ground will remain unsaturated because the air above this interface will be dry.

The interaction between cloud dynamics and cloud microphysical processes determines cloud droplet nucleation in cumulus clouds. Cloud top has long been a focus of the study of the evolution of the structure of cumulus clouds in Eulerian models (Squires, 1958; Paluch, 1979; Grabowski, 1993; Stevens et al., 1996a; Vaillancourt et al., 1997; Zhao and Austin, 2005). Two-dimensional and three-dimensional 
numerical studies have demonstrated that vortex circulations at cloud top result in lateral entrainment and mixing in cumulus clouds (Brenguier and Grabowski, 1993; Carpenter et al., 1998a,b; Zhao and Austin, 2005). The presence of cloud droplet nucleation at the cloud-environment interface has also been found (Feingold et al., 1994; Kogan et al., 1994; Slawinska et al., 2011) in such models. The gradient force of perturbation pressures that is included in two- and three-dimensional models may play a role in cloud droplet nucleation at cloud top. Issues with spurious nucleation at cloud top (Stevens et al., 1996b; Morrison and Grabowski, 2008; Grabowski and Morrison, 2008) complicate the picture. Note that two- and three-dimensional models must involve perturbation pressure in all kinds of their simulations. To disentangle the process underlying the nucleation, we need to use a special Eulerian model (Sun et al., 2012) firstly, which can take account of perturbation pressure but which also can be used in a mode where perturbation pressure is ignored in order to understand the true causes of nucleation at cloud top. Our results provide evidence for nucleation resulting from the gradient force of perturbation pressure. We use the Weather Research and Forecast model (WRF) further to verify the presence of physical nucleation at the interface of cloud-clear air.

\section{Dynamical processes for cumulus cloud formation}

Both buoyancy and pressure perturbations dominate the evolution of cumulus clouds (List and Lozowski, 1970; Yau, 1979; Schlesinger, 1980; Zhao and Austin, 2005). The vertical motion of air is mainly determined by the pressure gradient force and the gravitational force. The basic state of the atmosphere assumes that these two forces balance each other to reach hydrostatic equilibrium. Any perturbations of pressure and temperature, defined as the deviation of the local value from that of the environment at the same height, can result in the formation of a perturbation pressure gradient force acting simultaneously with the gravitational and buoyancy forces on the perturbed air and its environmental air. The buoyancy of the air can be described as follows:

$B=\frac{\theta^{\prime}}{\theta_{0}}+0.61 q_{\mathrm{v}}^{\prime}$

where $\theta^{\prime}$ denotes the deviation of potential temperature from the basic state, $\theta_{0}$ represents the potential temperature of the basic state, and $q_{\mathrm{v}}^{\prime}$ denotes the deviation of mixing ratio of water vapor from the basic state.

The generation and propagation of convective motion in cumulus clouds are physically both caused by the gradient force of perturbation pressures and buoyancy. The perturbation pressure $\left(\pi^{\prime}\right)$ is composed of a perturbation pressure $\left(\pi_{\mathrm{b}}^{\prime}\right)$ due to local buoyancy, a drag-induced perturbation pressure $\left(\pi_{\text {drag }}^{\prime}\right)$ (once hydrometeors are produced) and a dynamic perturbation pressure $\left(\pi_{\mathrm{d}}^{\prime}\right)$ induced by air motion. Convec- tion may be initiated by a buoyancy force $(B g)$ and a perturbation pressure gradient force $\left(\frac{\partial \pi^{\prime} \mathrm{b}}{\partial z}\right)$. If we assume that the atmosphere is initially still hydrostatic $\left(\frac{\partial \pi^{\prime} \mathrm{b}}{\partial z}=B g\right)$, horizontal motion of the air will be produced due to the horizontal gradient force of pressure $\left(\pi=\pi_{\mathrm{e}}+\pi_{\mathrm{b}}^{\prime}, \pi_{\mathrm{e}}\right.$ is the hydrostatic environmental pressure). Meanwhile, because of air mass continuity, horizontal air movement will trigger vertical air movement. Simultaneously, a dynamic perturbation pressure $\left(\pi_{d}^{\prime}\right)$ field concomitant with the hydrodynamics of the motion field can be generated (List and Lozowski, 1970; Das, 1979) and in turn affect the convection field through the gradient force of pressure $\left(\pi=\pi_{\mathrm{e}}+\pi_{\mathrm{b}}^{\prime}+\pi_{d}^{\prime}\right)$. However, the vertically moving air must be initially driven by some vertical forces. Since the perturbed atmosphere is non-hydrostatic $\left(\frac{\partial \pi^{\prime} \mathrm{b}}{\partial z} \neq B g\right)$, the nonhydrostatic force $\left(\frac{\partial \pi^{\prime} \mathrm{b}}{\partial z}-B g\right)$ drives the perturbed air upwards. As a result, the gradient force of the dynamic perturbation pressure $\left(\frac{\partial \pi^{\prime}}{\partial z}\right)$ triggered by the upward air motion acts on the air above the perturbed air. Therefore, it is essential to take account of the pressure gradient force generated by perturbation pressures, in addition to thermal buoyancy force, in an investigation of cumulus cloud development.

\section{Simulation with perturbation pressures}

We carried out a numerical experiment to investigate cloud droplet nucleation with the ice phase omitted with the $1.5 \mathrm{D}$ model (Sun et al., 2012). The hygroscopic growth of cloud condensation nuclei and their redistribution in cloud droplet spectra due to collision and coalescence will be explicitly determined with bin microphysics. The detailed treatments can be found in the paper of Sun et al. (2012). Figure 1 shows spatial and temporal evolution of thermodynamic variables with perturbation pressures and with perturbation pressures omitted. We will first discuss the simulation results with perturbation pressures considered in these multiple figures before returning to look at the non-pressure perturbation run. Figure 1a shows the time variation of the vertical velocities and the liquid water content for the case in which condensation and evaporation are assumed to be the only microphysical processes. The dynamic structures of the simulated convection lead to the transportation of water vapor upward from low altitudes and the formation of cloud droplets. Since air motion obeys the mass continuity constraint, the vertical distributions of updraughts result in dynamic entrainment at the lower part of the convection, and dynamic detrainment at the upper part of the convection. Such horizontal air movement physically results from the gradient force of perturbation pressures. Upward motion is not limited to regions of saturation. In the developing stage, unsaturated air above cloud top moves upwards with small vertical velocities.

The pressure gradient force generated by the dynamic perturbation pressure is responsible for the upward movement 

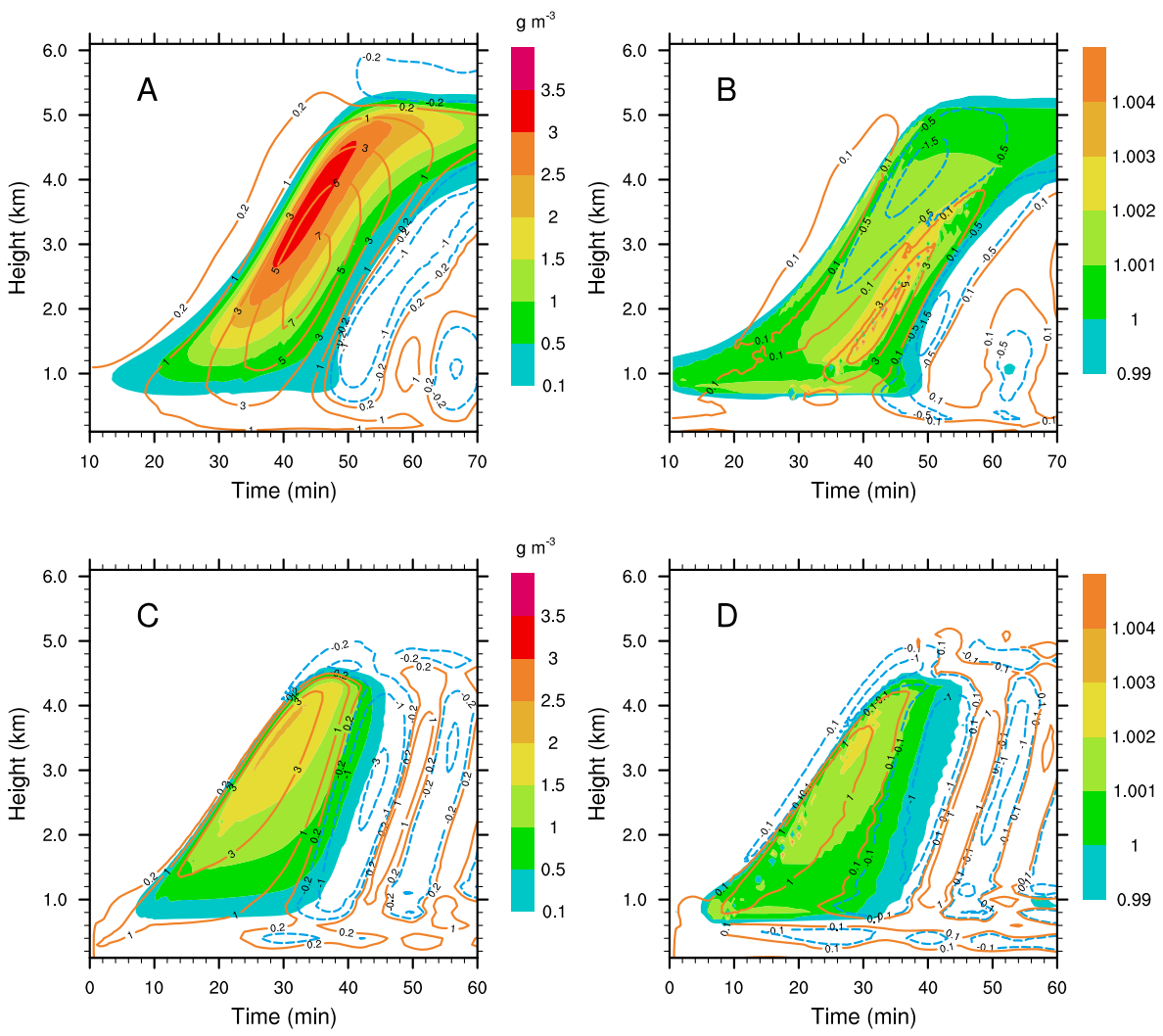

Fig. 1. Spatial and temporal evolution of thermodynamic variables with perturbation pressures $(\mathbf{A}, \mathbf{B})$ and with perturbation pressures omitted (C, D). (A) and (C) Liquid water content (in $\mathrm{g} \mathrm{m}^{-3}$ ) (shaded area) and vertical velocity $\left(\mathrm{m} \mathrm{s}^{-1}\right.$ ) (solid and dashed lines) as a function of time and height. (B) and (D) Supersaturation ratio and net vertical acceleration $d w / d t\left(\mathrm{~cm} \mathrm{~s}^{-2}\right)$ (solid and dashed lines) as a function of time and height.

of unsaturated air above the cloud. Figure $1 \mathrm{~b}$ illustrates the corresponding net vertical acceleration of the convection in a Lagrangian view. In the developing stage, positive vertical acceleration dominates the upper and lower parts of the convection. Positive vertical accelerations appear above cloud top and below cloud base, accelerating the transportation of unsaturated moist air upwards from low altitudes above the cloud top and moist air supply into the cloud from below the cloud base. The pressure gradient force generated by perturbation pressures dominates the contribution of the positive vertical acceleration in the upper region as both the thermal buoyant force and drag force oppose the perturbation pressure gradient force (Fig. 2a, b and c). In the lower part of the convection, both the buoyancy force and the pressure gradient force generated by the perturbation pressure contribute to the positive vertical acceleration. This result indicates that both forces play important roles in the generation and propagation of convection. We notice that thermal buoyancy reversal occurs at the cloud top (Fig. 2b). This is due to dry adiabatic ascent of unsaturated moist air, as observed by others in convection simulations (Schlesinger, 1980; Yau, 1980). The pressure gradient force generated by the dynamic perturbation pressure causes the initial vertical acceleration of the unsaturated moist air above the cloud top. The positive high centre of total perturbation pressure at the top of the convection (Fig. 2d) shows the conversion between dynamic perturbation pressure and kinetic energy (Yau, 1979). Simulations with three-dimensional models have shown that the dynamic perturbation pressure dominates at the upper part of the perturbation pressure centre (Schlesinger, 1984; Zhao and Austin, 2005). Therefore, the pressure gradient force induced by the dynamic perturbation pressure accelerates air parcels in the upper part, and decelerates air parcels in the lower part of the positive high centre, thus diminishing the gradient of vertical air velocity. The vertical velocity distribution determines the locations of newly activated cloud droplets that originate at the cloud summit and the cloud base. Correspondingly, the liquid water content first increases with height and then decreases with height (Fig. 1a). Two peak values of supersaturation appear, one at the cloud base and the other at cloud top (Fig. 1b). The latter one manifests as a $100 \mathrm{~m}$ thick band of elevated supersaturation that occurs at the cloud top between 25 and $50 \mathrm{~min}$, which indicates the occurrence of expansion cooling processes at the interfaces of cloud-environment air. The peak value at the cloud base has always been found in parcel modelling studies 

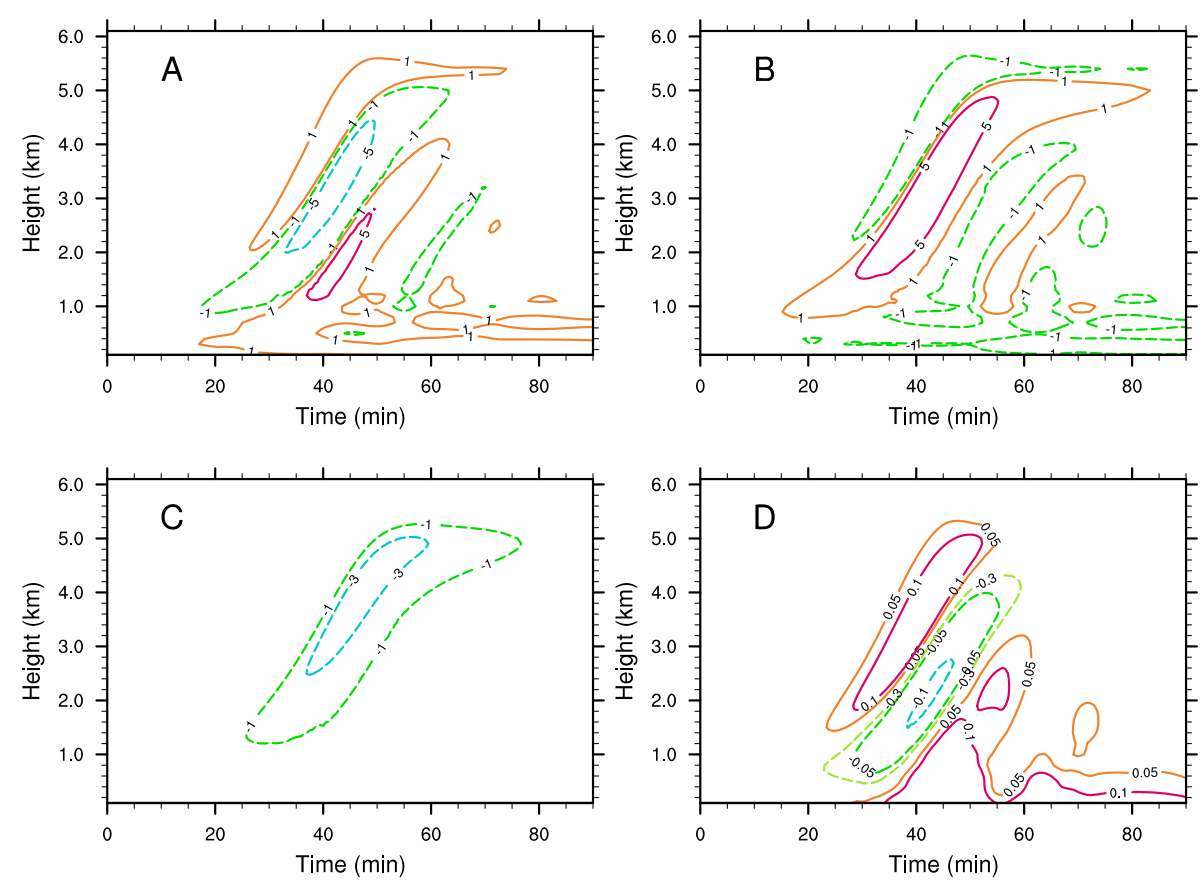

Fig. 2. Spatial and temporal evolution of forces and pressure deviation. (A) Gradient force generated by perturbation pressures per unit mass $\left(\mathrm{cm} \mathrm{s}^{-2}\right)$, (B) Thermal buoyancy force per unit mass $\left(\mathrm{cm} \mathrm{s}^{-2}\right),(\mathbf{C})$ Drag force per unit mass $\left(\mathrm{cm} \mathrm{s}^{-2}\right)$ and (D) Pressure deviation $(m b)$ as a function of time and height.

(Beard and Ochs, 1993; Segal et al., 2003, 2007). Because the relative humidity gradient at cloud top is small, the relative humidity being greater than $98 \%$ in most unsaturated grid boxes immediately above the saturated grid boxes at the cloud summit in the developing stage (see Fig. 1b), the supersaturation that develops cannot be ascribed to being spurious as in the numerical experiment without the dynamic field (Stevens et al., 1996b).

The previously reported spurious activation of cloud droplets in the cloud boundary grid boxes is due to the application of a grid-averaged field to determine the microphysical processes when the cloud interface crosses the grid boxes. However, evaporation near cloud edge and overshoots of supersaturation inside the cloud are only pronounced when there is a high gradient of relative humidity between the cloud grid boxes and the adjacent cloudfree grid boxes (Stevens et al., 1996b). Moreover, if spurious activation occurred in the present simulation in the cloudboundary grid boxes, the cloud droplet spectra would be composed of new spuriously activated small cloud droplets and advected large cloud droplets in the same grid boxes. As a result, the cloud spectra in the cloud-boundary grid boxes would be significantly broadened. Since saturation ratio is only a function of temperature, pressure and water vapour mixing ratio, the temperature change due to excess evaporation and excess condensation can be eliminated by our modified time-splitting numerical method which can prevent overevaporation and over-condensation. We used this algorithm for microphysical processes in all our simulations. If excessive cooling by evaporation or excessive heating by condensation occurs in the grid boxes a smaller time step is used to recalculate the diffusion growth of cloud droplets. Also the original state of saturation for all grid boxes is kept unchanged during condensation and evaporation, which means that evaporation will be stopped when the cloud boundary grid boxes become saturated in the next time step and condensation will be stopped when cloud grid boxes become undersaturated in the next time step. In other words, the saturation state of grid boxes can only be changed by the expansion cooling or compression heating in such a scenario.

Figure $3 \mathrm{a}$ shows the vertical variations of the size distributions of unactivated aerosol particles of ammonium sulfate and of cloud droplets $37 \mathrm{~min}$ into the simulation. The cloud consists of two components characterized by the different vertical variation of the average diameter of the droplets. In the upper part of the cloud, the average sizes of cloud droplets with a single-peaked distribution decrease with increasing altitude, which is obviously different from what would happen due to spurious nucleation, as a result of the continuous activation of cloud droplets at the cloud-clear air interface and their subsequent condensational growth. In the lower part of the cloud, average sizes of cloud droplets increase with increasing altitude due to condensational growth of the activated ammonium sulfate particles entering the cloud from its base and lower lateral sides. Between these two regions, the cloud droplet spectra are broadened and 

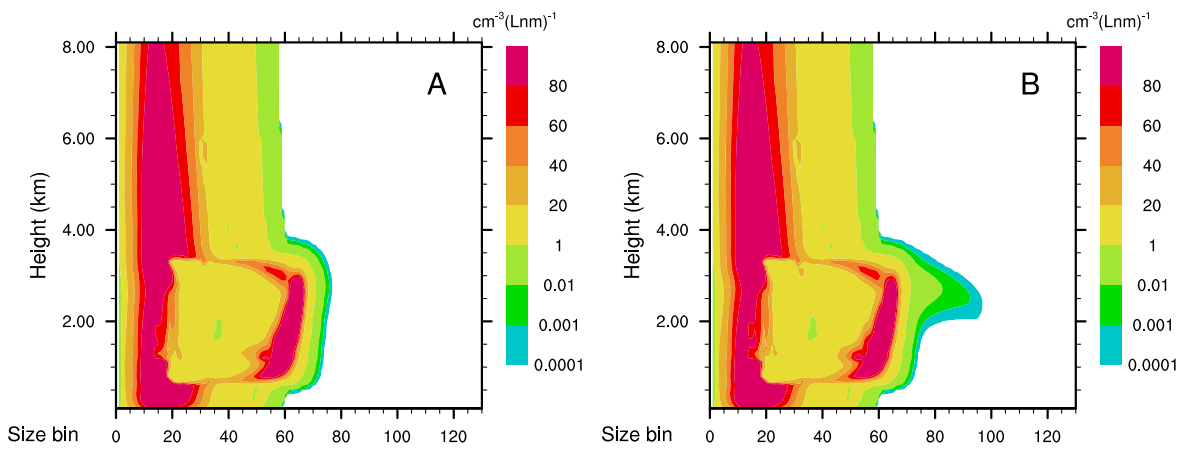

Diameter $(\mu \mathrm{m}) \quad 0.14 \quad 1.41 \quad 14.3 \quad 144.01450 .01 .46 \mathrm{e} 4$

Diameter $(\mu \mathrm{m}) \quad 0.14 \quad 1.41 \quad 14.3 \quad 144.01450 .01 .46 \mathrm{e} 4$
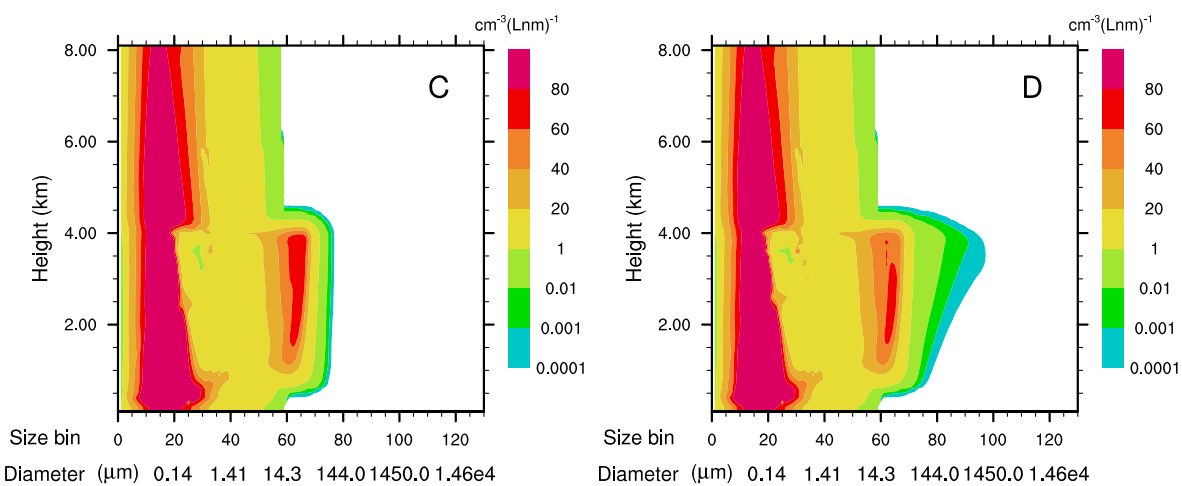

Fig. 3. Number distributions of unactivated ammonium sulfate aerosol particles and of water droplets as a function of height, natural logarithm of the water mass of the bin and natural logarithm of the aerosol mass of the bin $f\left(\mathrm{Lnm}_{\text {water }}, \mathrm{Lnm}_{\text {aerosol }}, Z\right)\left(\mathrm{Number}^{-3}\left(\mathrm{Lnm}_{\mathrm{Water}}\right)^{-1}\right)$ at $37 \mathrm{~min}$ in simulations with perturbation pressures (A, B) and at $31 \mathrm{~min}$ in simulations with perturbation pressures omitted (C, D). (A) and (C) Simulations of condensation and evaporation. (B) and (D) Simulations of condensation and evaporation as well as collision and coalescence.

bimodal due to vertical eddy exchanges. In a second simulation that includes collision and coalescence in addition to droplet condensational growth (Fig. 3b), large cloud droplets are found in the middle of the cloud air.

\section{Simulation without perturbation pressures}

To evaluate the impacts of perturbation pressures on cloud droplet nucleation, we repeated the previous simulation with perturbation pressures omitted. The simulation results show that the cloud top and cloud base are characterized by stronger and weaker gradients of thermodynamic properties, respectively, compared with those from the simulation with perturbation pressures. For example, vertical velocities gradually increase with height and then decrease sharply at the cloud top (Fig. 1c), in contrast to the simulation with perturbation pressures included. This scenario results in a strong air divergence at the cloud top and a weak air convergence at the cloud base in order to obey the air continuity constraint. The cloud free air above the cloud top moves horizontally away from the cloud path with extraordinary speeds when the cloud air moves upwards. Note that horizontal air movement is simply diagnosed from the air continuity equation.
The thermal buoyant force is the only driving force to act on the cloud air in this scenario. In the developing stage, positive vertical acceleration dominates the places in which condensation occurs while negative vertical acceleration dominates the cloud-top interface in which evaporation occurs. The weakened moist air supply at the cloud base and the lack of ascending moist air above the cloud top lead to a less vigorous cloud than the cloud simulated with perturbation pressures.

We noticed that there is still a supersaturation band at the cloud base, and above this band the saturation ratio generally increases with the height due to the acceleration of the vertical velocity (Fig. 1d). However, the supersaturation band at the cloud-top boundary has vanished, which means that cloud droplets are not activated at the cloud-clear air interface. Figure $3 \mathrm{c}$ and $\mathrm{d}$ show the number distribution of unactivated ammonium sulfate aerosol particles and of cloud droplets with the collision and coalescence processes omitted and with these processes considered. The average sizes of the cloud droplets increase with height, which is consistent with results performed with parcel models (Pruppacher and Klett, 1997; Segal et al., 2003, 2007). The concentration of cloud droplets also increase with height due to additional activation because of the acceleration of the vertical 
velocity (Fig. 3c). Large cloud droplets appear at the cloud summit (Fig. 3d). The concentration of cloud droplets is less than that of the simulation with pressure perturbations for the same concentration and size distribution of ammonium sulfate aerosols. We noticed that there are no cloud droplets with average sizes decreasing with height in the upper part of the cloud as in the simulation including pressure perturbations. This result further indicates that the gradient force of the dynamic perturbation pressure is responsible for the cloud droplet activation at the cloud-environment interface at the cloud summit.

\section{Comparisons with a three-dimensional model}

We used a simplified anelastic $1.5 \mathrm{D}$ cylindrical model for investigation of the cloud spectral broadening at the cloud top. However, more complex three-dimensional models can further elucidate the processes that we described here. For instance, Zhao and Austin (2005) showed that there is a vortexlike circulation at the cloud top in the late developing stage of cumulus clouds, which in turn impacts the droplet spectral evolution. However, the impacts of the vortex-like circulation at the cloud top in the early developing stage of cumulus cloud formation are still unknown. Furthermore, the horizontal distribution of vertical velocities (assumed to be sinusoidal in our simulations (Sun et al., 2012)) can increase the horizontal vorticity of air flow in the vertical plane, and strengthen the pressure gradient force of the dynamic perturbation pressure compared with a top-hat distribution of vertical velocities. Since the different horizontal distribution of vertical velocities in real cumulus clouds can strengthen or weaken this force, three dimensional models with bin microphysics are needed to study this impact further.

\subsection{Model description}

To study the early stage of cumulus cloud formation, experiments are performed using an LES version of the Advanced Research WRF (ARW) model (Version 3.3.1) (Skamarock et al., 2008) with a new treatment of aerosol-cloud interactions based on a bin method. The WRF model is a compressible and nonhydrostatic model. We coupled our bin microphysics with the ARW dynamics solver to investigate the cloud spectral broadening. Aerosol activation and cloud droplet condensation and evaporation are the only microphysical processes considered in the microphysics solver, which is based on solving the kinetic equation system for the size distribution of aerosol particles and cloud droplets. The approach yielding a doubling of the particle mass after two grid cells is used for the mass grid coordinate with total 60 mass bins for each particle class. The radius of particles is from $0.008 \mu \mathrm{m}$ to $7.130 \mu \mathrm{m}$ for aerosol particles and from $0.084 \mu \mathrm{m}$ to $80.67 \mu \mathrm{m}$ for cloud droplets. Aerosol particles are also assumed to be composed of fully soluble ammonium sulfate. According to the method of Pruppacher and Klett (1997), aerosol activation is treated by calculating the minimum activation radius of dry aerosol particles under the supersaturation diagnosed from the temperature and water vapor fields. Aerosol particles exceeding the critical size will be activated. If the critical diameter of water droplets is less than that of the minimum size bin of cloud droplets the new cloud droplets will be put into the lowest size bin of cloud droplets. Unlike the aerosol treatment in the 1.5D model (Sun et al., 2012), aerosol regeneration is ignored when evaporation occurs and aerosol mass distributions in cloud droplet spectra are not followed.

Simulations are performed in a $12.3 \times 12.3 \times 5.025 \mathrm{~km}^{3}$ domain with a relatively fine grid resolution, $50 \mathrm{~m}$ in the horizontal and $25 \mathrm{~m}$ in the vertical. The time step is $2 \mathrm{~s}$ for the simulation. The time-splitting procedure of the $1.5 \mathrm{D}$ model is applied for microphysical processes.

\subsection{Initial condition}

We used the same convection case as the simulations with the $1.5 \mathrm{D}$ cylindrical model under ammonium sulfate concentration of $C_{3}$ (Sun et al., 2012). The ambient wind is also ignored in this experiment. The convection is initiated by a moist thermal bubble as was the trigger function (Equation (2)) in WRF model. The horizontal radius of the perturbation is $3000 \mathrm{~m}$, and the vertical radius of the perturbation is 400 $m$. The maximum perturbation temperature is $1.0^{\circ} \mathrm{C}$.

$\theta^{\prime}=1.0 \times \cos ^{2}\left(\frac{\pi}{2} \cdot \sqrt{\frac{x^{2}}{3000^{2}}+\frac{y^{2}}{3000^{2}}+\frac{z^{2}}{400^{2}}}\right)$

where $x, y$ and $z$ are the coordinates of a perturbation grid point with the vertical axis at the central domain and with the horizontal axes at $400 \mathrm{~m}$ above the ground.

\subsection{Results}

Figure 4a shows the vertical cross section of the vertical velocity and the liquid water content at $5.5 \mathrm{~min}$. The updraft is surrounded by a weak axisymmetric downdraft. The upward movement of the cloud air is not coincident with that of the moist air. The cloud movement lags behind the updraft. The vertical variation of the liquid water shows a parabolic trend, which means that the liquid water content increases with height first and then decreases with height. A vortexlike structure of the wind field appears in the simulation (Fig. 4b). However, the entrainment of unsaturated air occurs only at the bottom of the cloud rather than the lateral cloud boundaries in the early stage of cumulus cloud formation. The low liquid water content at the cloud summit cannot be explained by the entrainment mechanism for the simulation. We noticed that two supersaturation bands occur at the cloud top (Fig. 4b), one supersaturation band appears at the cloud-clear air interface and another one is just behind the first one. The first one manifests as a $25 \mathrm{~m}$ thick band 

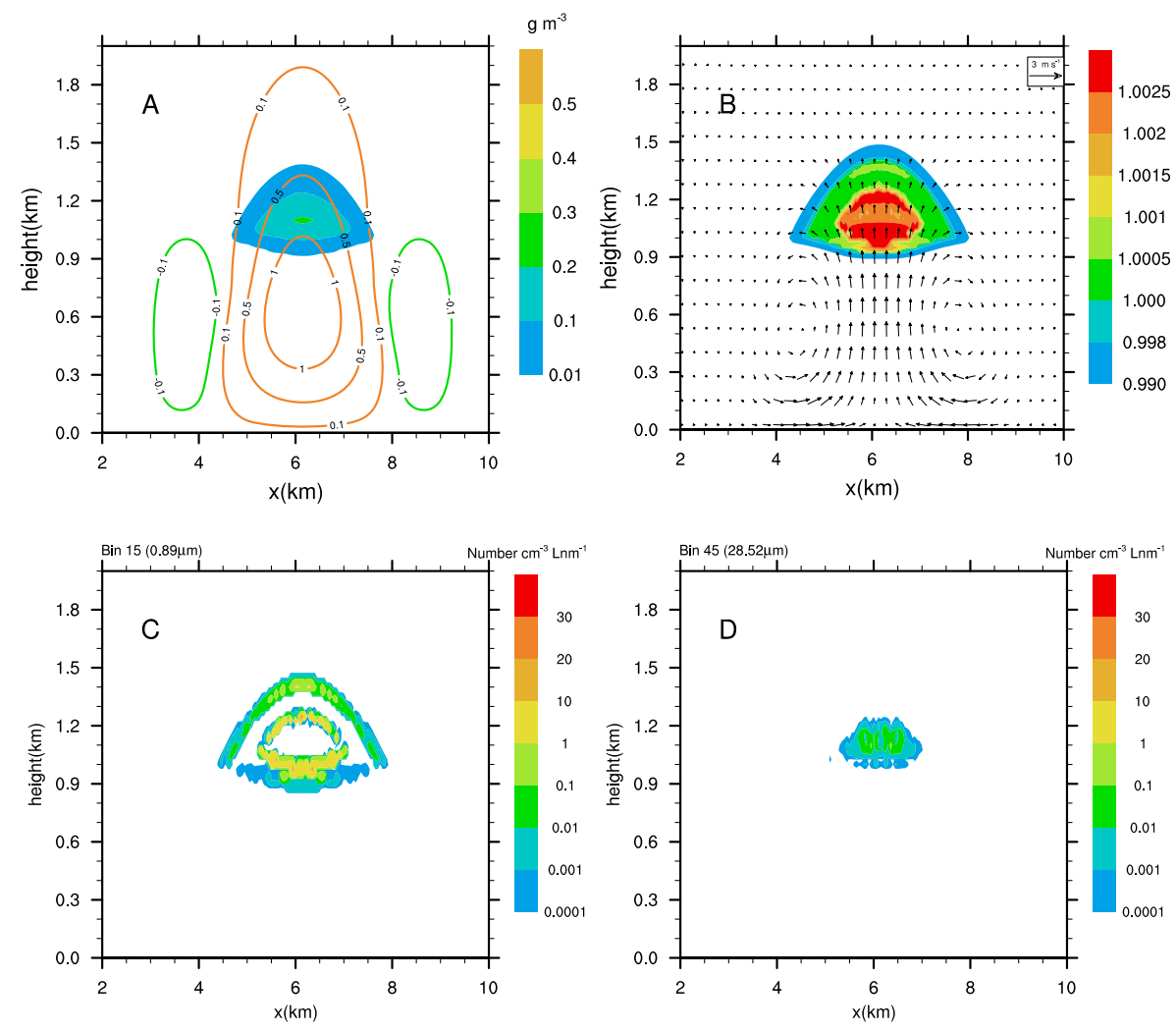

Fig. 4. The vertical cross section of the simulation at $5.5 \mathrm{~min}$. (A) Vertical velocity $\left(\mathrm{m} \mathrm{s}^{-1}\right.$ ) (solid line) and liquid water content (in $\mathrm{g} \mathrm{m}^{-3}$ ) (shaded area), (B) Wind field and saturation ratio, (C) The number density of cloud droplets $f\left(\operatorname{Lnm}_{\mathrm{water}}, X, Z\right)\left(\mathrm{Number}^{-3}\right.$ $\left.\left(\mathrm{Lnm}_{\text {water }}\right)^{-1}\right)$ in 15 bin $(0.89 \mu \mathrm{m})$, (D) In 45 bin $(28.52 \mu \mathrm{m})$.

of elevated supersaturation that occurs at the cloud top horizontally between 5.5 and $6.5 \mathrm{~km}$. Similarly, there are two supersaturation bands at the cloud base.

The temporal and spatial evolution of the saturation ratio indicates that the parabolic feature of the liquid water content in the vertical direction is due to the new activation of cloud droplets at the cloud boundaries. The upward movement of moist air above the cloud boundaries is responsible for the new activation of cloud droplets at the cloud top. The expansion cooling of upward movement may initially result in one layer of the most nearly saturated moist air becoming saturated. The level of initial cloud droplet activation has been defined as the lifting condensation level (LCL). If the layer of moist air above the LCL is also nearly saturated it will eventually become saturated due to its upward movement. All saturation ratios in the grid boxes immediately above the saturated grid boxes are greater than 0.99 (Fig. 4b). Therefore, the cloud boundaries in the developing stage of cumulus cloud formation should be saturated. As a result, condensation rather than evaporation occurs at the cloud boundary at cloud top. The continuous spatial variation of saturation ratio in the cloud boundary at the cloud top actually reflects the property of air continuity. The saturation change should be spatially continuous for upward moving moist air. The spa- tial distribution of cloud droplet spectra further indicates that activation of new cloud droplets occurs at the cloud boundaries. The vertical size variation of cloud droplets also shows a parabolic trend (Fig. $4 \mathrm{c}$ and d). The initially activated cloud droplets grow into large cloud droplets at the center of the cloud (Fig. 4d). The nearly newly activated cloud droplets are dominant at the boundaries of the cloud (Fig. 4a). Since the acceleration of the vertical velocity of cloud air can lead to an increase of its supersaturation, two secondary supersaturation bands are formed following the first supersaturation bands (Fig. 4b). Correspondingly, two secondary activated cloud droplet bands occur in the internal cloud (Fig. 4c).

Comparing the simulations with the 1.5D model, we find that the vertical gradient of vertical velocity at the cloud top is diminished in the early stage. As a result, the weakened horizontal divergence at the cloud top results in more cloud droplets being activated at the upper boundaries. Therefore, the simulations with the simplified two-cylinder model may underestimate the cloud droplet activation at the cloud summit in the early developing stage of cumulus cloud formation. This underestimation may be due not only to the limitations of the 1.5D model itself, but also the anelastic approximation since density fluctuations associated with perturbation pressures are ignored in such an approximation (Durran, 1988; 
Sun et al., 2012). Vertical convergence at the cloud top locally increases air density and the positive perturbation pressures in fully compressible air. The larger gradient force of perturbation pressures than that with the anelastic approximation will act on the cloud free air above the cloud top.

\section{Conclusions}

The pressure gradient force generated by the dynamic perturbation pressure can cause spectral broadening of cumulus cloud droplets at the cloud top. The appearance of small cloud droplets at the cloud-clear air interface is supported by observations of small cloud droplets at cloud top (Blyth and Latham, 1985; Paluch and Baumgardner, 1989; Stith, 1992). The observed small cloud droplets at the cloud top in the developing stage of cumulus clouds should be formed by primary nucleation rather than by secondary nucleation or by evaporation. In a simulation in which buoyancy is the only driving force for the development of the cloud we found that a high gradient of relative humidity occurs at the cloud top boundary but spurious nucleation of cloud droplets can be prohibited by our modified time-splitting method. The gradient force of perturbation pressures plays a role in cloud droplet nucleation at the cloud-environment interface in the simulation with perturbation pressures considered. The simulation with the three dimensional model verifies that cloud top is also the source of cloud droplets in the developing stage.

The above finding can explain why the observed liquid water content and the temperature of cumulus clouds in the early stage are less than the adiabatic values of air parcels ascending from the cloud base. The cloud volumes in the upper part of cumulus clouds result from moist air being forced by the pressure gradient force generated by the dynamic perturbation pressure and then ascending from different altitudes above the cloud base. As a result, the upper parts of cumulus clouds contain lower liquid water contents than those of adiabatic values of cloud air ascending from the cloud base.

The decrease in liquid water content with height at the upper parts of cumulus clouds has often been observed (Pruppacher and Klett, 1997; Rauber et al., 2007; Heymsfield et al., 2009). Entrainment of unsaturated air into clouds and subsequent cloud dilution have been applied to explain this phenomenon by means of boundary mixing either from the interface of the cloud top (Squires, 1958; Paluch, 1979) or from the periphery of the main updraught with vortex-like structures in the upper parts of cumulus clouds (Grabowski, 1993; Carpenter et al., 1998a,b; Zhao and Austin, 2005). However, our study shows that this phenomenon need not be always explained by entrainment and mixing because cloud air adiabatically ascending from different lifting condensation levels can also lead to a decrease of liquid water content with height.
The existence of small cloud droplets near cumulus cloud edges has always been considered as a result of homogeneous mixing of dry environmental air (Warner, 1969, 1973; Baker and Latham, 1979; Small and Chuang, 2008) and subsequent cloud droplet evaporation (Telford et al., 1984). Cloud droplet nucleation instead of droplet evaporation can also result in the formation of small cloud droplets at the cloudenvironment interface. Furthermore, the entrained air from a subsiding cloudy shell in the late development stage of cumulus clouds will be driven upwards by the pressure gradient force generated by the dynamic perturbation pressure (Zhao and Austin, 2005). This expansion cooling process can also lead to entrained air to be supersaturated. The numerical simulations for deep convective clouds showed that small droplet nucleation occurs at the cloud periphery (Khain and Pokrovsky, 2004; Slawinska et al., 2011). The main physical mechanism for small cloud droplet formation may be the nucleation process rather than the evaporation process in the developing stage of cumulus clouds. Therefore, the phenomenon of cloud droplet spectrum broadening toward small sizes may be mainly attributed to dynamically driven microphysical processes of cloud lateral-interface nucleation, cloud top-interface nucleation and even in-cloud nucleation (Pinsky and Khain, 2002; Segal et al., 2003; Prabha et al., 2011; Khain et al., 2012).

The formation of sufficiently large cloud droplets (diameter around or greater than $50 \mu \mathrm{m}$ ) is key to the initiation of precipitation (Beard and Ochs, 1993). Inhomogeneous entrainment of unsaturated air into clouds (inhomogeneous mixing) (Baker et al., 1980) has been considered as one of important physical mechanisms to form collisioncoalescence initiators (Small and Chuang, 2008). However, entrainment and mixing cannot occur in the early developing stage of cumulus clouds. The role of inhomogeneous mixing in large cloud droplet formation may be overestimated.

The above evidences suggest that cloud droplet nucleation should be a dominant physical process of cumulus clouds in their developing stage while cloud droplet evaporation should be a dominant physical process in their dissipating stage under consideration of their dynamic fields. Therefore, the spatial and temporal evolutions for both cloud liquid water contents and cloud droplet spectra should be highly related to interface nucleation rather than interface evaporation in the developing stage of cumulus clouds.

Moreover, cumulus cloud development depends on the vertical profile of temperature, but it should be emphasized that the vertical profile of relative humidity is also an important factor that impacts cumulus cloud formation. The relative humidity above the cloud base impacts on both cloud droplet nucleation and cloud droplet evaporation. 
Acknowledgements. We appreciate financial support from the Natural Science and Engineering Research Council of Canada (NSERC), CMAM, Centre for Climate and Global Change Research, and financial support from Institute of Atmospheric Physics, Chinese Academy of Sciences for "100 Talents program of Chinese Academy of Sciences".

Edited by: M. King

\section{References}

Baker, M. B. and Latham, J.: The evolution of droplet spectra and the rate of production of embryonic raindrops in small cumulus clouds, J. Atmos. Sci., 36, 1612-1615, 1979.

Baker, M. B., Corbin, R. G., and Latham, J.: The influence of entrainment on the evolution of cloud droplet spectra: I. A model of inhomogeneous mixing, Q. J. Roy. Meteor. Soc., 106, 581-598, 1980.

Beard, K. V. and Ochs, H. T.: Warm-rain initiation: An overview of microphysical mechanisms, J. Appl. Meteor., 32, 608-625, 1993.

Blyth, A. M. and Latham, J.: An airborne study of vertical structure and microphysical variability within a small cumulus, Q. J. Roy. Meteor. Soc., 111, 773-792, 1985.

Brenguier, J. E. and Grabowski, W. W.: Cumulus entrainment and cloud droplet spectra: A numerical model within a twodimensional dynamical framework, J. Atmos. Sci., 50, 120-136, 1993.

Carpenter, R. L., Droegemeier, J. K. K., and Blyth, A. M.: Entrainment and detrainment in numerically simulated cumulus congestus clouds. Part I: General results, J. Atmos. Sci., 55, 3417-3432, 1998a.

Carpenter, R. L., Droegemeier, J. K. K., and Blyth, A. M.: Entrainment and detrainment in numerically simulated cumulus congestus clouds. Part III: Parcel analysis, J. Atmos. Sci., 55, 34403455, 1998b.

Das, P.: A non-Archimedian approach to the equations of convection dynamics, J. Atmos. Sci., 36, 2183-2190, 1979.

Durran, D. R.: Improving the anelatstic approximation, J. Atmos. Sci., 46, 1453-1461, 1988.

Feingold, G., Stevens, B., Cotton, W. R., and Walko, R. L.: An explicit cloud microphysics/LES model designed to simulate the Twomey effect, Atmos. Res., 33, 207-233, 1994.

Grabowski, W. W.: Cumulus entrainment, fine-scale mixing and buoyancy reversal, Q. J. Roy. Meteor. Soc., 119, 856-935, 1993.

Grabowski, W. W. and Morrison, H.: Toward the mitigation of spurious cloud-edge supersaturation in cloud models, Mon. Weather Rev., 136, 1224-1234, 2008.

Heymsfield, A. J., Bansemer, A., Heymsfield, G., and Fierro, A. O.: Microphysics of maritime tropical convective updrafts at temperatures from $-20^{\circ} \mathrm{C}$ to $-60^{\circ} \mathrm{C}$, J. Atmos. Sci., 66, 3530-3562, 2009.

Khain, A. and Pokrovsky, A.: A simulation of effects of atmospheric aerosols on deep turbulent concective clouds using a spectral microphysics mixed-phase cumulus cloud model. Part II: Sensitivity study, J. Atmos. Sci., 61, 2983-3001, 2004.

Khain, A. P., Phillips, V., Benmoshe, N., and Pokrovsky, A.: The role of small soluble aerosols in the microphysics of deep maritime clouds, J. Atmos. Sci., 69, 2787-2807, 2012.
Kogan, Y. L., Lilly, D. K., Kogan, Z. N., and Filyushkin, V. V.: The effect of $\mathrm{CCN}$ regeneration on the evolution of stratocumulus cloud layers, Atmos. Res., 33, 137-150, 1994.

List, R. and Lozowski, E. P.: Pressure perturbation and buoyancy in convective clouds, J. Atmos. Sci., 27, 168-170, 1970.

Morrison, H. and Grabowski, W. W.: Modeling supersaturation and subgrid-scale mixing with two-moment bulk warm microphysics, J. Atmos. Sci., 65, 792-812, 2008.

Paluch, I. R.: The entrainment mechanism in Colorado cumuli, J. Atmos. Sci., 36, 2467-2478, 1979.

Paluch, I. R. and Baumgardner, D. G.: Entrainment and fine-scale mixing in a continental convective cloud, J. Atmos. Sci., 46, 261278, 1989.

Pinsky, M. and Khain, A. P.: Effects of in-cloud nucleation and turbulence on droplet spectrum formation in cumulus clouds, Q. J. Roy. Meteor. Soc., 128, 1-33, 2002.

Prabha, T., Khain, A. P., Goswami, B. N., Pandithurai, G., Maheshkumar, R. S., and Kulkarni, J. R.: Microphysics of premonsoon and monsoon clouds as seen from in-situ measurements during CAIPEEX, J. Atmos. Sci., 68, 1882-1901, 2011.

Pruppacher, P. S. and Klett, J. D.: Microphysics of clouds and Precipitation, Kluwer Academic, Dordrecht, The Netherlands, 1997.

Rauber, R. M., Stevens, B., Ochs III, H. T., Knight, C., Albrecht, B. A., Blyth, A. M., Fairall, C. W., Jensen, J. B., Lasher-trapp, S. G., Mayol-Bracero, O. L., Vali, G., Anderson, J. R., Baker, B. A., Bandy, A. R., Burnet, E., Brenguier, J.-L., Brewer, W. A., Brown, P. R. A., Chuang, P., Cotton, W. R., Girolamo, L., Geerts, B., Gerber, H., Goke, S., Gomes, L., Heikes, B. G., Hudson, J. G., Kollias, P., Lawson, R. P., Krueger, S. K., Lenschow, D. H., L. Nuijens, D. W. O., Rilling, R. A., Rogers, D. C., Siebesma, A. P., Snodgrass, E., Stith, J. L., Thornton, D. C., Tucker, S., Twohy, C. H., and Zuidema, P.: Rain in shallow cumulus over the ocean: The RICO campaign, B. Am. Meteor. Soc., 88, 19121928, 2007.

Schlesinger, R. E.: A three-dimensional numerical model of an isolated thunderstorm. Part II: Dynamics of updraft splitting and mesovortex couplet evolution, J. Atmos. Sci., 37, 395-420, 1980.

Schlesinger, R. E.: Effects of the pressure perturbation field in numerical models of unidirectionally sheared thunderstorm convection: Two versus three dimensions, J. Atmos. Sci., 41, 15711587, 1984.

Segal, Y., Pinsky, M., Khain, A., and Erlick, C.: Thermodynamic factors influencing bimodal spectrum formation in cumulus clouds, Atmos. Res., 66, 43-64, 2003.

Segal, Y., Pinsky, M., and Khain, A.: The role of competition effect in the raindrop formation, Atmos. Res., 83, 106-118, 2007.

Skamarock, W. C., Klemp, J. B., Dudhia, J., Gill, D. O., Barker, D. M., Huang, X. Y., Wang, W., and Powers, J. G.: A description of the advanced research WRF version 3, NCAR Tech. Rep. NCAR/TNC475+STR, 2008.

Slawinska, J., Grabowski, W. W., Pawlowska, H., and Morrison, H.: Droplet activation and mixing in large-eddy simulation of a shallow cumulus field, J. Atmos. Sci., 69, 444-462, 2011.

Small, J. D. and Chuang, P. Y.: New observations of precipitation initiation in warm cumulus clouds, J. Atmos. Sci., 65, 29722982, 2008.

Squires, P.: Penetrative downdraughts in cumuli, Tellus, 10, 381389, 1958. 
Stevens, B., Feingold, G., Cotton, W. R., and Walko, R. L.: Elements of the microphysical structure of numerically simulated nonprecipitating stratocumulus, J. Atmos. Sci., 53, 980-1007, 1996a.

Stevens, B., Walko, R. L., Cotton, W. R., and Feingold, G.: The spurious production of cloud-edge supersaturations by Eulerian models, Mon. Weather Rev., 124, 1034-1041, 1996 b.

Stith, J. L.: Observations of cloud-top entrainment in cumuli, J. Atmos. Sci., 47, 1334-1347, 1992.

Sun, J., Ariya, P. A., Leighton, H. G., and Yau, M. K.: Modelling study of ice formation in warm-based precipitating shallow cumulus clouds, J. Atmos. Sci., 69, 3315-3335, 2012.

Telford, J. W., Keck, T. S., and Chai, S. K.: Entrainment at cloud tops and the droplet spectra, J. Atmos. Sci., 41, 3170-3179, 1984.

Vaillancourt, P. A., Yau, M. K., and Grabowski, W. W.: Upshear and Downshear Evolution of Cloud Structure and Spectral Properties, J. Atmos. Sci., 54, 1203-1217, 1997.
Warner, J.: The microstructure of cumulus cloud. Part I. General features of the droplet spectrum, J. Atmos. Sci., 26, 1049-1059, 1969.

Warner, J.: The microstructure of cumulus cloud. Part IV: The effect on the droplet spectrum of mixing between cloud and environment, J. Atmos. Sci., 30, 256-261, 1973.

Yau, M. K.: Perturbation pressure and cumulus convection, J. Atmos. Sci., 36, 690-694, 1979.

Yau, M. K.: A two-cylinder model of cumulus cells and its application in computing cumulus transports, J. Atmos. Sci., 37, 2470$2485,1980$.

Zhao, M. and Austin, P. H.: Life cycle of numerically simulated shallow cumulus clouds, J. Atmos. Sci., 62, 1291-1310, 2005. 